constipation but their relative clinical performance is unclear. Our aim was to investigate the diagnostic yield and clinical outcomes of DFP and ARM in chronic constipation.

Methods Patients who had undergone both DFP and ARM over a 3year period were studied retrospectively. Demographics, treatment and clinical outcomes were recorded. The diagnosis was recorded as "mixed" if investigation showed evidence of both anismus and anatomical problems such as rectocoele, intussusception or prolapse. The clinical outcome was defined as positive if the test resulted in treatment with symptomatic improvement, or resolution at followup. To determine whether there was a selection bias in those undergoing both DFP and ARM we additionally looked at the two groups having solely DFP or ARM from the same period.

Results DFP and ARM group: 43 patients (40 female, 58\% surgical referrals; age range 17-85 years; median 46) underwent both DFP and ARM. The diagnostic yield for DFP was higher at $98 \%$ (anismus $44 \%$, anatomical $40 \%$, mixed $14 \%$; normal $2 \%$ ) vs $47 \%$ for ARM (anismus 26\%, mixed 21\%; normal 53\%). There was diagnostic concordance in only 11 (26\%), partial concordance in $9(21 \%)$ and discordance in 23 (53\%) patients. Although the diagnostic yield of DFP was much greater than ARM in this combined group, both tests led to similar positive outcomes regardless (47\% in DFP vs $45 \%$ in ARM) when tests revealed a pathology. Single investigation groups: 10 patients had DFP alone (8 female, 60\% surgical referrals; age range $22-73$ years, median 55 ) with a diagnostic yield of $90 \%$ (anismus $30 \%$, anatomical $50 \%$, mixed $10 \%$; normal 10\%). The positive outcome in those with a detectable pathology was $33 \% .15$ patients had ARM alone (14 female, $27 \%$ surgical referrals; age range 19-75 years, median 50) with a diagnostic yield of 67\% (anismus; $33 \%$ normal). The positive outcome in those with a detectable pathology was $70 \%$.

Conclusion DFP had a higher diagnostic yield than ARM, but concordance was poor. Greater diagnostic yield did not translate into more positive clinical outcomes either. The clinical impact of additional DFP-based diagnoses is therefore questionable. The single test cohort data suggest that patients having DFP alone are a different clinical population from those who accessed both tests, since diagnostic yields and clinical outcomes were higher for ARM alone. The latter group were predominantly medical gastroenterology referrals. Further study is required to design optimal investigation strategies for chronic constipation.

Competing interests None declared.

\section{PWE-007 PARACRINE PROSTAGLANDIN-E SIGNALLING MODULATES CANINE GASTRIC EPITHELIAL CELL MIGRATION}

doi:10.1136/gutjnl-2012-302514d.7

${ }^{1} \mathrm{R}$ Hollins, ${ }^{*}{ }^{2} \mathrm{~A}$ I Freeman, ${ }^{3} \mathrm{P}$ J Cripps, ${ }^{3} \mathrm{~A} D$ Radford, ${ }^{1} \mathrm{P}$ J M Noble. ${ }^{1}$ Institute of Translational Medicine, University of Liverpool, Liverpool, UK; ${ }^{2}$ School of Veterinary Science, University of Liverpool, Liverpool, UK; ${ }^{3}$ Institute of Infection and Global Health, University of Liverpool, Liverpool, UK

Introduction Gastric ulceration is a limiting complication of therapy with cyclooxygenase (COX) antagonists, widely used anti-inflammatory/analgesic drugs in both humans and dogs. COX-derived prostaglandin E (PGE) has an important role in gastric defense and cytoprotection via promotion of blood flow and mucus secretion and inhibition of gastric acid secretion. Given the importance of gastric epithelial cell migration in reestablishing epithelial integrity following gastric damage, we have investigated whether paracrine PGE signalling has a role in the modulation of gastric epithelial cell migration.

Methods In order to retain paracrine signalling between different cell types, we isolated intact gastric glands via collagenase digestion of canine gastric mucosal tissue. Isolated glands spread in vitro to form islands of cells. The rate of gland spreading over $48 \mathrm{~h}$ was measured as a surrogate for cell migration speed. Lamellipodia protrusion was analysed as an index of spreading activity. A value for lamellopodia area was calculated by measuring spread area minus area bounded by nuclei of cells at the edge of spread glands. Spread glands, when serum-starved, exhibit a reduction in area. We added both a selective and a non-selective COX antagonist and $\mathrm{PGE}_{2}$ to serumstarved glands to assess their effects on migration. All treatments were added blindly to eliminate bias. Statistical significance was assessed using univariate analysis of variance. Expression of COX-2 and PGE receptors (EP-3 and EP-4) was assessed by RT-PCR and immunohistochemistry.

Results RT-PCR confirmed COX-2, EP-3 and EP-4 expression in our samples. COX-2 immunoreactivity was present in the majority of gland cells. The COX $1 / 2$ antagonist indomethacin $(50 \mu \mathrm{M})$ decreased spreading ( 0.85 -fold, $\mathrm{p}<0.05, \mathrm{n}=5-9$ for all experiments) and lamellipodia area (0.3-fold, $\mathrm{p}<0.05)$. The COX-2 selective antagonist NS-398 $(10 \mu \mathrm{M})$ caused similar decreases to indomethacin (0.8-fold and 0.65-fold respectively, $\mathrm{p}<0.05)$. PGE $(1 \mu \mathrm{M})$ prevented a 0.7 -fold reduction in island area elicited by incubation in serum free medium $(p<0.05)$.

Conclusion This data shows a role for COX-2 derived PGE in the promotion of gastric cell migration and cellular lamellipodia formation. A reduction of mucosal PGE via COX-2 antagonism may therefore inhibit gastric epithelial cell migration contributing to COX-antagonist elicited gastric ulceration in both humans and dogs.

Competing interests None declared.

\section{Oesophageal II

PWE-008 DO STATINS PREVENT THE HISTOLOGICAL SUBTYPES OF
OESOPHAGEAL CANCER? PROSPECTIVE DATA FROM THE
UK GENERAL PRACTICE RESEARCH DATABASE (GPRD)

doi:10.1136/gutjnl-2012-302514d.8

${ }^{1} \mathrm{H}$ Y Bhutta, ${ }^{2,3}$ L Alexandre, ${ }^{*} \mathrm{~A}$ Clark, ${ }^{4} \mathrm{~S}$ Holt,,${ }^{1,3} \mathrm{M}$ Lewis, ${ }^{2,3} \mathrm{~A}$ Hart. ${ }^{1}$ Department of General Surgery, Norfolk and Norwich Hospital, Norwich, UK; ${ }^{2}$ Department of Gastroenterology, Norfolk and Norwich Hospital, Norwich, UK; ${ }^{3}$ Norwich Medical School, University of East Anglia, Norwich, UK; ${ }^{4}$ Roundwell Medical Centre, Norwich, UK

Introduction The incidence of oesophageal adenocarcinoma (OAC) has risen dramatically in the Western world and is associated with a poor prognosis. Statins show anti-cancer properties in experimental work with OAC cell lines for example reduced cell proliferation, increased apoptosis. This study aimed to investigate if statins are negatively associated with the development of two different histological subtypes of oesophageal cancer, OAC and oesophageal squamous cell cancer (OSCC), in a prospective cohort study.

Methods The cohort was over 4 million people in the General Practice Research Database (GPRD), a UK database of 488 nationwide general practices. Information is recorded on medication use prior to development of other illnesses, including cancers. Statin use was defined as a prescription for a minimum of 10 months preceding diagnosis of oesophageal cancer. Approximately half the GP practices in the GPRD are linked to the NHS cancer registry, allowing identification and sub-classification of histologically confirmed cases of OAC and OSCC. Each case was matched with four controls and conditional logistic regression estimated the OR plus $95 \% \mathrm{CIs}$ for the development of each type of cancer, adjusted for diabetes, BMI, smoking, aspirin, PPIs and drugs that relax the lower oesophageal sphincter.

Results 581 histologically confirmed cases of OAC (77.8\% men, mean age 70.7 years, $\mathrm{SD}=11.4)$ and 332 cases of $\mathrm{OSCC}(38.6 \%$ men, 
71.9 years, $\mathrm{SD}=12.3$ ) were identified between 2000 and 2008. The median length of statin use in cases and controls prior to diagnosis or index date was 3.9 years (IOR 2.3-6.1 years). Analysis by histological subtype showed an inverse association for OAC (OR $0.61,95 \%$ CI 0.39 to 0.94 ) and for OSCC (OR 0.41, 95\% CI 0.21 to $0.80)$. Categorisation into lipophilic and hydrophilic statins demonstrated significant inverse associations for only lipophilic statins for both OAC (OR 0.60, 95\% CI 0.38 to 0.95 ) and OSCC (OR $0.46,95 \%$ CI 0.23 to 0.91$)$. There was a dose-response effect for statins and both OAC and OSCC $(p<0.05$ for linear trend) comparing high and low-dose statin use.

Conclusion The data supports lipophilic statins having a protective effect against the development of both OAC and OSCC, with evidence of a dose-response trend. Confirmation in other populations is required. Detailed statin use, according to class and dose, should be measured in future aetiological studies of both OAC and OSCC. The information supports assessment of these drugs in chemoprevention of OAC in the general population and those with Barrett's oesophagus in clinical trials.

Competing interests None declared.

\section{PWE-009 DIETARY DOCOSAHEXAENOIC ACID IS INVERSELY ASSOCIATED WITH THE RISK OF DEVELOPING OESOPHAGEAL ADENOCARCINOMA: A UK PROSPECTIVE STUDY IN THE EPIC-NORFOLK COHORT, USING INFORMATION FROM 7-DAY FOOD DIARIES}

doi:10.1136/gutjnl-2012-302514d.9

\begin{abstract}
${ }^{1,2} \mathrm{~L}$ Alexandre, ${ }^{*} 1,2 \mathrm{M}$ Yates, ${ }^{3} \mathrm{R}$ Luben, ${ }^{2} \mathrm{E}$ Cheong, ${ }^{2} \mathrm{~L}$ Igali, ${ }^{4} \mathrm{R}$ Fitzgerald, ${ }^{3} \mathrm{~K}-\mathrm{T}$ Khaw, ${ }^{1,2} \mathrm{~A}$ Hart. ${ }^{1}$ Norwich Medical School, University of East Anglia, Norwich, UK; ${ }^{2}$ Department of Gastroenterology, Norfolk and Norwich University Hospital, Norwich, UK; ${ }^{3}$ Strangeways Research Laboratory, University of Cambridge, Cambridge, UK; ${ }^{4}$ Medical Research Council Epidemiology Unit, University of Cambridge, Cambridge, UK
\end{abstract}

Introduction Dietary docosahexaenoic acid (DHA) and eicosapentanaenoic acid (EPA) are both $n-3$ polyunsaturated fatty acids (n3 PUFAs) which have both anti-inflammatory and anti-carcinogenic actions, including inhibiting angiogenesis and tumour cell proliferation, and promoting apoptosis. The aim of this study was to conduct the first epidemiological investigation to determine if there is an inverse association between DHA and EPA intake and the risk of developing both Barrett's oesophagus (BO) and oesophageal adenocarcinoma (OAC).

Methods A total of 25639 men and women aged 40-75 years, were recruited between the years 1993 and 1997 into the European Prospective Investigation into Cancer (EPIC)-Norfolk cohort Study. At baseline, participants completed detailed 7-day food diaries which were then coded by nutritionists. Subjects were then followed-up over subsequent years for the development of $\mathrm{BO}$ and OAC. A review of case notes confirmed these diagnoses. Dietary intake of n3 PUFAs were compared between cases and a random sample of 3797 controls in a case-cohort analysis. Intakes were divided into quintiles and both biological gradients and threshold effects were calculated. Cox regression estimated the HR for both DHA and EPA, adjusted for age, gender, BMI, smoking, alcohol, and arachidonic acid intake.

Results During follow-up, 104 patients were diagnosed with BO (80\% men, median age 67.0 yrs [IOR 61.1-73.1] at diagnosis) after a median follow-up of 6.2 yrs (IOR 4.1-8.1). A further 63 patients developed OAC (83\% men, median age 73.0 yrs [IOR 67.0-78.0] at diagnosis) after a median follow-up of 6.4 yrs (IOR 4.4-8.9). For OAC, there was a large significant inverse association, in a threshold manner, comparing the lowest quintile with a summation of the top four quintiles of dietary intake, for DHA (HR 0.47, 95\% CI 0.25 to $0.88, \mathrm{p}=0.02$ ). EPA (HR 0.60, 95\% CI 0.31 to $1.15, \mathrm{p}=0.12$ ) intake was not significantly associated with $\mathrm{OAC}$. For $\mathrm{BO}$, no significant associations were found with either dietary DHA (HR 0.69, 95\% CI 0.40 to $1.20, p=0.19$ ) or EPA (HR $1.03,95 \%$ CI 0.56 to 1.90 , $\mathrm{p}=0.93$ ). No gradient effects across quintiles were seen for either $\mathrm{BO}$ or OAC

Conclusion Dietary DHA was associated with an approximate $50 \%$ risk reduction for $\mathrm{OAC}$, although there were no significant effects for $\mathrm{BO}$. The data support a role for dietary DHA in preventing the malignant transformation of $\mathrm{BO}$ to $\mathrm{OAC}$, and therefore should be measured in future aetiological studies of OAC.

Competing interests None declared.

\section{PWE-010 DIETARY CAMPESTEROL IS INVERSELY ASSOCIATED WITH THE RISK OF DEVELOPING OESOPHAGEAL ADENOCARCINOMA: A UK PROSPECTIVE STUDY IN THE EPIC-NORFOLK COHORT, USING INFORMATION FROM 7-DAY FOOD DIARIES}

doi:10.1136/gutjnl-2012-302514d.10

1,2 L Alexandre, ${ }^{* 1,2} \mathrm{M}$ Yates, ${ }^{3} \mathrm{R}$ Luben, ${ }^{2} \mathrm{E}$ Cheong, ${ }^{2} \mathrm{~L}$ Igali, ${ }^{4} \mathrm{R}$ Fitzgerald, ${ }^{3} \mathrm{~K}-\mathrm{T}$ Khaw, ${ }^{1,2} \mathrm{~A}$ Hart. ${ }^{1}$ Norwich Medical School, University of East Anglia, Norwich, UK; ${ }^{2}$ Department of Gastroenterology, Norfolk and Norwich University Hospital, Norwich, UK; ${ }^{3}$ Strangeways Research Laboratory, University of Cambridge, Cambridge, UK; ${ }^{4}$ Medical Research Council Epidemiology Unit, University of Cambridge, Cambridge, UK

Introduction Dietary phytosterols (PS), including campesterol, the most bioavailable of this group, are structurally similar to cholesterol, and are present in grain legumes, cereals, nuts and vegetable oils. Experimental studies have shown PS have several anticarcinogenic effects, including inducing apoptosis and inhibiting both angiogenesis and cell proliferation. The aim of this study was to conduct the first epidemiological investigation to determine if an inverse association exists between PS intake and the risk of both Barrett's oesophagus (BO) and oesophageal adenocarcinoma (OAC). Methods A total of 25639 men and women aged $40-75$ years, were recruited between the years 1993 and 1997 into the European Prospective Investigation into Cancer (EPIC)-Norfolk cohort Study. At baseline, participants completed detailed 7-day food diaries which were coded by nutritionists. Subjects were followed-up over subsequent years for the development of $\mathrm{BO}$ and $\mathrm{OAC}$. A review of case notes confirmed these diagnoses. Dietary intakes were compared between cases and a random sample of 3797 controls in a case-cohort analysis. Cox regression estimated the HR for both campesterol and total PS.

Results During follow-up, 104 patients were diagnosed with BO (80\% men, median age 67.0 yrs [IOR 61.1-73.1] at diagnosis) after a median follow-up of 6.2 yrs (IOR 4.1-8.1). A further 63 patients developed OAC (83\% men, median age 73.0 yrs [IOR 67.0-78.0] at diagnosis) after a median follow-up of $6.4 \mathrm{yrs}$ (IOR 4.4-8.9). For $\mathrm{BO}$, no significant associations were found with campesterol (HR $1.47,95 \%$ CI 0.77 to 2.79 ) or total dietary PS (HR $1.28,95 \%$ CI 0.70 to 2.33), in either sex. For OAC, in men, there were inverse associations with campesterol (HR $0.43,95 \%$ CI 0.22 to $0.83, p=0.01$ ), in a threshold manner, comparing the lowest with a summation of the top four quintiles of intake. In women, for OAC, there were no such associations with campesterol (HR 2.13,95\% CI 0.44 to 10.15 , $\mathrm{p}=0.34$ ). Total PS intake (HR $0.71,95 \%$ CI 0.38 to 1.35 ) was not significantly associated with $\mathrm{OAC}$ in either sex.

Conclusion Campesterol intake was associated with an approximate $55 \%$ risk reduction for $\mathrm{OAC}$ in men, although there were no significant effects in $\mathrm{BO}$ or for either condition in women. The data support a role for dietary campesterol, in preventing the malignant transformation of $\mathrm{BO}$ to $\mathrm{OAC}$, and therefore these micronutrients should be measured in future aetiological studies of OAC.

Competing interests None declared. 